\title{
Simulation and Characterization of Junction Less CMOS Inverter at Various Technology Nodes
}

\author{
Vishal Narula', Charu Narula ${ }^{2}$ and Jatinder Singh ${ }^{2}$ \\ 'Lovely Professional University, Phagwar-144411, Punjab, India; \\ Vishal.20405@|pu.co.in \\ ${ }^{2}$ University Institute of Engineering Technology, Panjab University, Chandigarh - 160014, Punjab, India; \\ charu_uiet@pu.ac.in, singh01_jatinder@yahoo.com
}

\begin{abstract}
The simulation and drawing based on new type of technology namely junction less transistor technology CMOS inverter is discussed in this paper at various channel lengths. The transient curve, noise margin, various differences between conventional and junction less technology has been illustrated in this paper. The surface conduction and bulk conduction steps using visual TCAD is also expressed. The designing and fabrication steps along with the pros and cons have been characterized. The short channel parameter on which all other parameters directly or indirectly depends is calculated for both conventional and junction less transistor at different nodes. The noise margin and propagation delay at channel length $10 \mathrm{~nm}, 20 \mathrm{~nm}, 30 \mathrm{~nm}$ and $40 \mathrm{~nm}$ is calculated using TCAD simulation software. It has been found that CMOS inverter is giving best results when made using junction less technology.
\end{abstract}

Keywords: CMOS, Junction Less Transistor (JLT), Noise margin, TCAD

\section{Introduction}

Digital integrated circuits are mostly consisting of transistors. In digital market it is observed that physical size of the devices is getting chopped with the enhancement of technology. In year 1971, semiconductor devices were manufactured at $10 \mathrm{um}$ nodes and now at present this technology node has reached to approximately $20 \mathrm{~nm}$ and the devices are getting portable ${ }^{2}$. As physical size of device is getting lower means the size of internal components is also reducing. Transistor acts as a backbone in any electrical circuit. In order to achieve a very proficient performance, it is very important for internal components to work at their best If we look upon the MOS transistor, short channel effects comes into picture when device comes to scaling and therefore there is a need to improve the performance of internal components of the device to achieve performance far better than the threshold. Internal electrical components need to provide a high quality output with lesser losses of power. MOS transistor act as an electrical switch which functions in many circuits like complementary MOS etc. Switching is one of the most significant activities in any circuitt ${ }^{-3}$

The conventional MOSFET comprises of source and drain junction. The short channel effects which devastate the performance of any device are involved is due to presence of $\mathrm{P}-\mathrm{N}$ junctions. It has become a need to reduce short channel effects along with the reduction in channel of the devicef. It has also become a challenge for fabrication industry to fabricate such conventional MOSFET's at smaller scale with very fine and accurate alignment of junctions. A new concept has been enlightened to reduce such complexity in which MOS transistor are made without junctions resulting into reduction of short channel effects known as junction less technology. A junction less device has a uniform doping throughout the distance from source to drain ${ }^{4-6,7,9}$

Electron densities varying with gate to source voltage for conventional and junction less dual gate MOS is shown in Figure 1 and Figure 2 respectively. In figure 1 we can see the various stages of electron density of conventional MOS at different gate voltages. When gate voltage is at $0 \mathrm{~V}$

*Author for correspondence 
there are no carriers in the channel region, as gate voltage starts increasing and reaches at $1 \mathrm{~V}$ we can see the channel starts forming at upper and lower surface of the substrate due to the presence of dual gate. After gate voltage increased from $1 \mathrm{~V}$ we can imagine the proper inversion of channel taking place in conventional dual gate MOS. A conventional MOS device works after inversion mode is setup. The channel so created in conventional mode is on the surface of the device called surface conduction and in junction less channel is created in the bulk of the device called as bulk conduction

In dual gate MOSFET, there are two gates which are interconnected to each other and controlling the flow of carriers whether it is conventional or junction less device. The work function of both the gates in junction less device plays an efficient role to switch off or on the device. Junction less devices are always operated at those work functions which are opposite to conventional one. This means the work function for conventional NMOS is same as that for junction less PMOS2. In Figure 2. we can see the channel formation in terms of electron density when gate voltages are increased from $0 \mathrm{~V}$ to $1 \mathrm{~V}$.

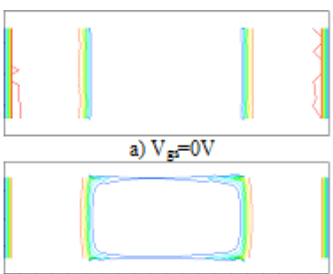

c) $\mathrm{V}_{\mathrm{g}}=0.5 \mathrm{~V}$

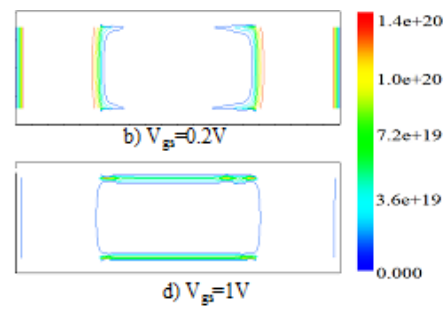

Figure 1. (a-d). Showing electron densities of conventional

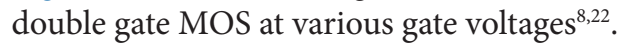
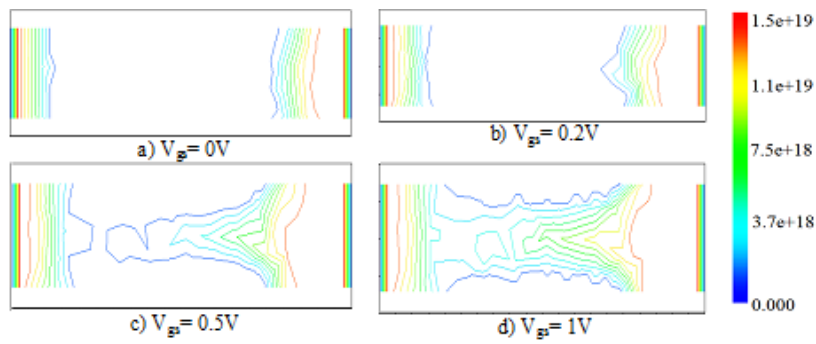

Figure 2. (a-d). Showing electron densities of Junction less double gate MOS at various gate voltages 2 .

It can be observed that electron density starts increasing from the bulk of the device. Hence conduction in junction less device is called as bulk conduction. There is no inversion mode which is present in such devices due to the presence of one type of carrier in the entire device. The reduction in fabrication steps injunction less devices is an attractive feature. As a result fabrication complexity will also get reduced while manufacturing small scale devices. This is all possible because of the absence of source and drain junctions. To achieve an accurate doping profile while manufacturing the $\mathrm{P}-\mathrm{N}$ junctions in conventional devices is a difficult task. The physics of both the type of transistor is different but electrical characteristics follow the same increasing/decreasing order 7

In this paper the drawing structure and simulations of double gate junction less and conventional $\mathrm{P}$ and $\mathrm{N}$-type along with short channel and performance parameters have been measured at different channel lengths. In addition to this the structure and various simulation results of junction less CMOS inverter is also evaluated at various technology nodes. All structures N-type DGJLT, P-type DGJLT, N-type Conventional DGMOS, P-type conventional DGMOS, junction-less CMOS inverter are drawn using TCAD simulator. All simulations are performed at different dimensions, different work function, different channel lengths.

This paper is classified as follows. In section 2, the structure of various devices and conditions for simulations will be discussed. In section 3, the simulations results of junction less CMOS and other conventional and junction less DGNMOS and DGPMOS will be discussed at different parameters and in section 4 will conclude all the discussion.

\section{Device Structure}

The devices used for simulation are drawn using TCAD software 2 . All structures are drawn at different channel lengths. The conventional N-type and P-type double gate MOS are drawn with N-type polysilicon and P-type Polysilicon as gate materials respectively. The N-type and P-type junction less double gate MOS have P-type polysilicon and N-type polysilicon respectively. The structure of junction-less N-type/P-type and conventional N-type/P-type are shown in Figure 3. a and 3. b respectively. The conventional double gate $\mathrm{N}$-type/Ptype MOS has different source/drain and channel doping whereas in junction-less N-type/ P-type has an identical doping. 


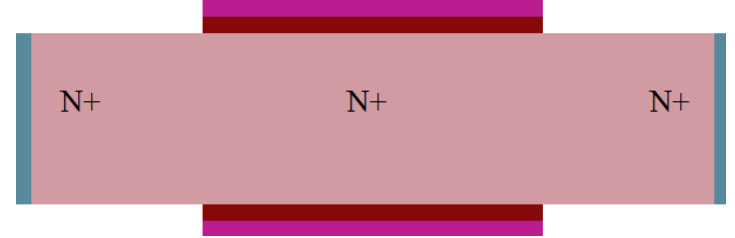

Figure 3. a) showing junction less Double gate MOS with uniform donor impurity throughout from source to channel to drain resulting into $\mathrm{N}$-type structure.

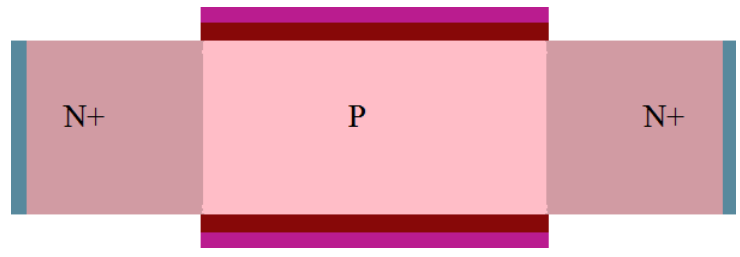

Figure 3. b) showing Conventional Double gate MOS with source and drain doped with donor impurity and substrate as acceptor resulting into N-type structure.

A new type of device made using junction less technology called junction less double gate complementary MOS/ A junction less inverter is made by combining both junction less NMOS and PMOS with an oxide separating them. All design parameters are same used for designing JLT PMOS/NMOS. Design parameter values are illustrated in Table A. CMOS inverter is used in almost all digital circuits for switching

This JLT CMOS made using double gate transistors are easily fabricated as there is no need to form n-well/Pwell which is necessary for conventional CMOS. As in this type of inverter we have both junction less transistors giving their best performance parametric values at small dimensions. This type of inverter can be easily fabricated at smaller scale dimensions. There are no junctions present in this CMOS structure which will reduce the devastation of the performance of the inverter. The physics of JLT CMOS inverter is different due to presence of bulk conduction in this technology. CMOS acts as an inverter in which the input is applied onto both the gates of the transistor and output is obtained at the drain ends of both the transistor. The width of JLT PMOS is made double than NMOS so that charging time of output node can become almost same as that of discharging time p $^{\text {. }}$.

The doping for both junction less double gate NMOS and junction-less double gate PMOS are taken same as $1.5 \mathrm{e}+19 \mathrm{~cm}^{-3}$. This is due to series connection of both PMOS and NMOS The current flowing has to be same throughout the inverter. JLT CMOS inverter is structured by combining 10, 20,30,40nm of JLT-PMOS and NMOS. JLT CMOS structure is shown in Figure 4. Channel formation in JLT CMOS inverter at different gate voltages is also shown in Figure 5. This CMOS structure is closely packed than conventional one. Doping in junction less devices needs to be higher to achieve better on current due to the presence of one type of impurities 1 . All four gates in the JLT CMOS inverter structure are interconnected and input is applied to the common gate. Also both drains of PMOS and NMOS are too interconnected to obtain the output.

As JLT CMOS structure is made by combining both JLT PMOS and NMOS. Therefore it can be observed that channel formation which is used to carry current in the inverter is like one after the other. It can be seen in figure $\mathrm{E}$ that net increase in charge is observed first in JLT NMOS and then JLT PMOS. Clearly for JLT NMOS gate voltages will be applied positive as threshold voltage

Table A. Different parameter values used for simulation and design of structurese-21

\begin{tabular}{|c|c|c|c|c|c|c|}
\hline Parameter Name & $\begin{array}{c}\text { Conv. } \\
\text { DGNMOS }\end{array}$ & $\begin{array}{c}\text { JLT } \\
\text { DGNMOS }\end{array}$ & $\begin{array}{c}\text { Conv. } \\
\text { DGPMOS }\end{array}$ & $\begin{array}{c}\text { JLT } \\
\text { DGPMOS } \\
\end{array}$ & \multicolumn{2}{|c|}{ JLT CMOS } \\
\hline \multirow[t]{2}{*}{ Work function } & \multirow[t]{2}{*}{$4.2 \mathrm{eV}$} & \multirow[t]{2}{*}{$5.3 \mathrm{eV}$} & \multirow[t]{2}{*}{$5.2 \mathrm{eV}$} & \multirow[t]{2}{*}{$4.2 \mathrm{eV}$} & PMOS & NMOS \\
\hline & & & & & $4.2 \mathrm{eV}$ & $5.3 \mathrm{eV}$ \\
\hline Channel length & $10-40 \mathrm{~nm}$ & $10-40 \mathrm{~nm}$ & $10-40 \mathrm{~nm}$ & $10-40 \mathrm{~nm}$ & \multicolumn{2}{|c|}{$\begin{array}{l}\text { 10-40nm for both } \\
\text { PMOS and NMOS }\end{array}$} \\
\hline \multirow{2}{*}{$\begin{array}{l}\text { Type of gate } \\
\text { material }\end{array}$} & \multirow{2}{*}{$\begin{array}{l}\mathrm{N}+\text { polysil- } \\
\text { icon }\end{array}$} & \multirow{2}{*}{$\begin{array}{l}\mathrm{P}+\text { polysil- } \\
\text { icon }\end{array}$} & \multirow{2}{*}{$\begin{array}{c}\mathrm{P}+ \\
\text { polysilicon }\end{array}$} & \multirow{2}{*}{$\begin{array}{l}\mathrm{N}+\text { polysil- } \\
\text { icon }\end{array}$} & PMOS & NMOS \\
\hline & & & & & N+Poly & P+ Poly \\
\hline Oxide thickness & $1 \mathrm{~nm}$ & $1 \mathrm{~nm}$ & $1 \mathrm{~nm}$ & $1 \mathrm{~nm}$ & \multicolumn{2}{|c|}{$\begin{array}{l}1 \mathrm{~nm} \text { each for both } \\
\text { PMOS and NMOS }\end{array}$} \\
\hline Doping & $\begin{array}{l}\text { 1) N-type } \mathrm{S} / \mathrm{D} \\
=1 \mathrm{e}+20 \mathrm{~cm}^{-3} \\
\text { 2) P-type Sub } \\
=1 \mathrm{e}+18 \mathrm{~cm}^{-3}\end{array}$ & $\begin{array}{c}\text { N-type S/D/ } \\
\text { Sub: } 1.5 \mathrm{e}+19 \\
\mathrm{~cm}^{-3}\end{array}$ & $\begin{array}{l}\text { 1)P-type S/ } \\
\mathrm{D}=1 \mathrm{e}+20 \mathrm{~cm}^{-3} \\
\text { 2)N-type Sub- } \\
=1 \mathrm{e}+18 \mathrm{~cm}^{-3}\end{array}$ & $\begin{array}{c}\text { P-type S/D/ } \\
\text { Sub: } 1.5 \mathrm{e}+19 \\
\mathrm{~cm}^{-3}\end{array}$ & $\begin{array}{c}\text { Doping } \\
\text { for JLT I } \\
\text { JLT }\end{array}$ & $\begin{array}{l}\text { ues used } \\
\text { IOS and } \\
\text { LOS }\end{array}$ \\
\hline
\end{tabular}


for NMOS is positive and for JLT PMOS gate voltages are applied as negative.

In this paper Junction less CMOS inverter is made by using double gate MOS as there are two gates which are placed at the top and bottom to control the flow of carriers in both JLT PMOS and JLT NMOS. The presence of two gates in a structure also ensures that no part of the structure is far from the gate which is controlling the gate flow. Using double gate structure, it is possible to achieve better short channel effects ? $^{\text {? }}$. The design and fabrication of junction less CMOS is easier and simpler than conventional one. Doping and work function of both the JLT MOS is responsible for the current flowing in the device and is responsible for carriers to get depleted and hence making the device off

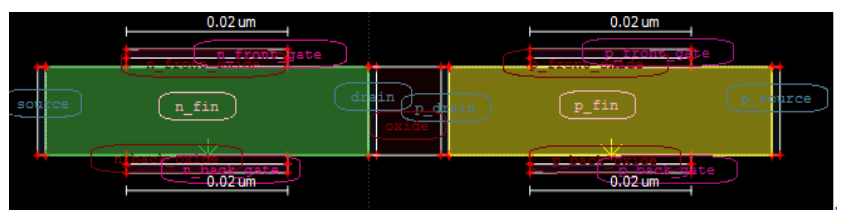

Figure 4. Structure of junction-less CMOS inverter月

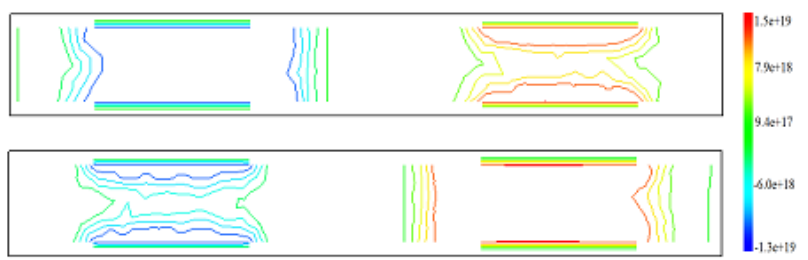

Figure 5. Net charge increase $\left(\mathrm{cm}^{-3}\right)$ in JLT PMOS after JLT NMOS in double gate Junction less CMOS inverter.

\section{Results and Comparison}

The performance of any structure depends upon the performance of individual components. As the CMOS inverter is made using PMOS and NMOS therefore the electrical characteristics of both types of transistor will be responsible for the results of an inverter. Conventional MOS and junction less MOS are compared at various channel lengths. There are different parameters which can define the performance of conventional and JLT namely ON current, OFF current, ON/OFF current ratio, Threshold voltage, subthreshold slope, Drain Induced Barrier Lowering (DIBL) are measured at various conditions mentioned in table $16,10-13$ All results of simulations are obtained after considering some conditions. There is no impact ionization effect is taken as due to this effect the carrier concentration will shoot up and will disturb all the analysis related to given parameters, mobility model is taken to be as Lombardi, all are performed at room temperature and at smaller voltages.

\subsection{Conventional DGMOS vs Junction Less DGMOS}

Conventional MOS are made of different types of doping in the substrate. They are namely $\mathrm{P}$ channel MOS or N channel MOS. Due to the presence of junctions it has been observed that short channel effects starts devastating the performance of the devicen. As technology nodes are reducing to improve the area and speed, it has been seen that leakage current which is an undesired current starts increasing to a large value and hence disturbing all performance parameters. Figure 6 shows the leakage current at 10, 20,30,40nm channel length of both conventional and junction less MOS. Junction less MOS are free of junctions and short channel effects are very small in comparison to conventional one. Leakage current is the most threatening parameter for any device. This can detrimental the accomplishment of any device 20,21.

From figure 6 it can be explained that the junction less transistor has a leakage current of order $10^{-9} \mathrm{~A}$ at $\mathrm{L}=10 \mathrm{~nm}$ and $10^{-15} \mathrm{~A}$ at $\mathrm{L}=40 \mathrm{~nm}$ and conventional double gate $\mathrm{MOS}$ has a leakage current of order $10^{-7} \mathrm{~A}$ at $\mathrm{L}=10 \mathrm{~nm}$ and $10^{-11}$ $A$ at $\mathrm{L}=40 \mathrm{~nm}$. The undesired current is decreasing as the length of the channel is increasing this is due to increase in the area of the device. Leakage current is responsible for the worst output. As technology nodes are decreasing year by year leakage current is increasing and in order to obtain a high class performance of the device along with the scaling, the junction less transistor technology has becoming an assurance to achieve the best performance parameters along with the scaling. The dimensions of the devices are lowered down to achieve the faster speed. But theleakage current as a tradeoff is spoiling the performance of the device. However it can be observed that for same conditions the conventional dual gate MOS is giving worst performance than junction less DGMOS. Also ON current is observed to be approximately of order $10^{-5} \mathrm{~A}$ for both types of technologies. All performance parameters such as ON current, OFF current, ON/OFF current ratio, Threshold voltage, subthreshold slope, Drain Induced Barrier Lowering (DIBL) at channel lengths $10 \mathrm{~nm}$, $20 \mathrm{~nm}, 30 \mathrm{~nm}, 40 \mathrm{~nm}$ have been calculated 2 . The leakage 
current in conventional double gate MOS is much higher than double gate Junction less transistor. Therefore the performance of any device made of conventional MOS will give worst performance than device made of junction less technology $15-17$.

\subsection{Junctionless CMOS inverter}

Complementary MOS technology is used in many digital circuitries. CMOS uses a combination of P-type and $\mathrm{N}$-type transistor. PMOS and NMOS are connected in series to achieve the same current ${ }^{19}$. It has been observed in previous section that conventional MOS has larger leakage current than junction less. Also fabrication of conventional MOS is somewhere typical than junction less MOS. To achieve best results from the device, it is necessary to use junction less based PMOS and NMOS. CMOS device has a main important characteristics that it has a good noise margin. Figure 7 shows the butterfly curve or voltage transfer characteristic curve of double gate junction less based CMOS inverter at channel lengths $10 \mathrm{~nm}, 20 \mathrm{~nm}, 30 \mathrm{~nm}, 40 \mathrm{~nm}$ of both PMOS and NMOS each.
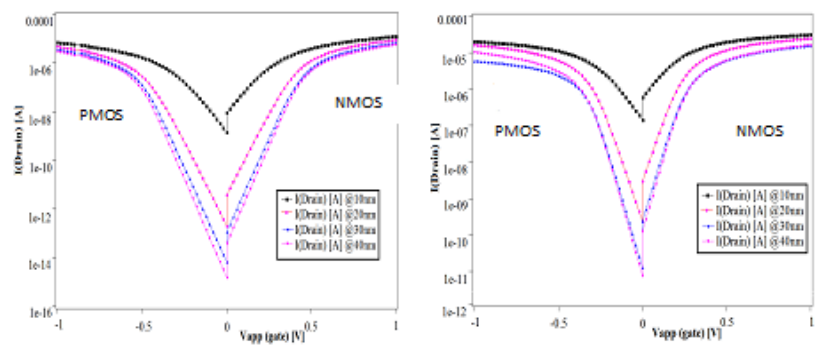

Figure 6. Transfer characteristics of double gate junction less (left) and conventional (right) $M O S$ at $\mathrm{V}_{\mathrm{ds}}=50 \mathrm{mV}$.

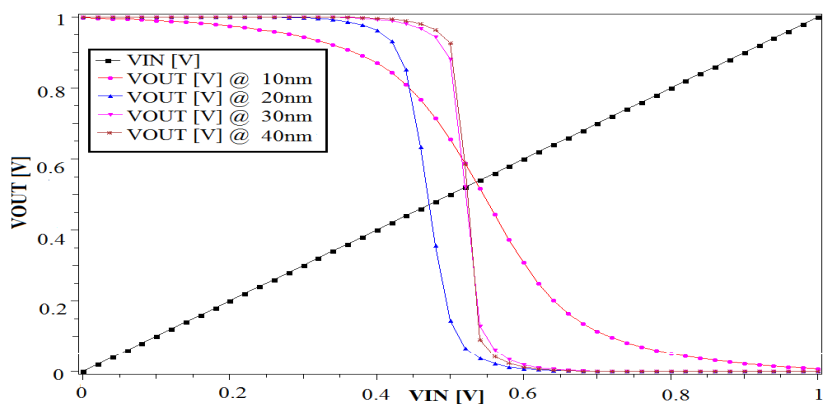

Figure 7. VTC curve of DG-junction less CMOS at various channel lengths.

It can be observed from the above figure that as the channel length is increased of both PMOS and NMOS say from $10 \mathrm{~nm}$ to $40 \mathrm{~nm}$, the VTC curve become more steeper. At $40 \mathrm{~nm}$ technology due to increase in area of the each device the leakage current is almost negligible and this means when the device is off there will almost no current which is flowing in the device and hence when input voltage becomes higher the output tends to zero immediately resulting into better performance of junction less based CMOS inverter. The below mentioned steps shows the fabrication of conventional CMOS inverter, it can be seen that total of 20 steps have to be followed for fabricating conventional CMOS 18

- Take a Substrate

- Grow an oxide layer over the substrate

- Grow a photoresist layer either positive or negative

- Do masking over it

- Remove the photoresist layer

- Remove the oxide layer deposited

- Again remove the photoresist layer depending upon where window has to be etched

- Using Ion- implementation, make $\mathrm{N}$-well regions

- Remove Silicon dioxide from selected areas

- Deposit the polysilicon layer on both the gates

- Remove unnecessary layers from the surface

- Grow the oxide layer again

- Do masking and diffuse N-type material to make Source and drain for NMOS

- Oxide layer which are deposited needs to be stripped

- Diffuse P-type material to form PMOS

- Again grow thick field oxide

- Do the metallization, using aluminium to form metal contacts

- Remove all the excess material from the surface

- Make the terminals

- Finally assigned all the names of the terminals In junction less transistor, there is no need to form any junctions in the entire device. Therefore no need to form $\mathrm{N}$-well and p-well regions mentioned in step no 8 . Along with this step, the other steps used to implement this region will also be reduced. So junction less based CMOS inverter will have lesser fabrication steps

Noise margin is defined as the amount of noise that CMOS circuit can handle without affecting the performance of the device. The VTC curve at channel length $40 \mathrm{~nm}$ is more stepper and better than any other channel length therefore noise margin is expected to achieve higher value. The value of noise margin is mentioned in Table B. 
Table B. Noise margin of junction less based CMOS inverter high voltage $1 \mathrm{~V}^{19}$

\begin{tabular}{lcc}
\hline Channel length & $\begin{array}{c}\text { High Noise Margin } \\
\left(\mathrm{NM}_{\mathrm{H}}\right)\end{array}$ & $\begin{array}{c}\text { Low Noise Margin } \\
\left(\mathrm{NM}_{\mathrm{I}}\right)\end{array}$ \\
\hline $10 \mathrm{~nm}$ & 0.28 & 0.32 \\
$40 \mathrm{~nm}$ & 0.43 & 0.52 \\
\hline
\end{tabular}

The transient response of junction less CMOS inverter is shown in Figure 8. Junction less technology can also show the same transient response than that of conventional in an improved version and with an easy fabrication. The transient response is an evidence which shows that junction less PMOS and NMOS are worth to make an important building block of electrical circuit ${ }^{10}$

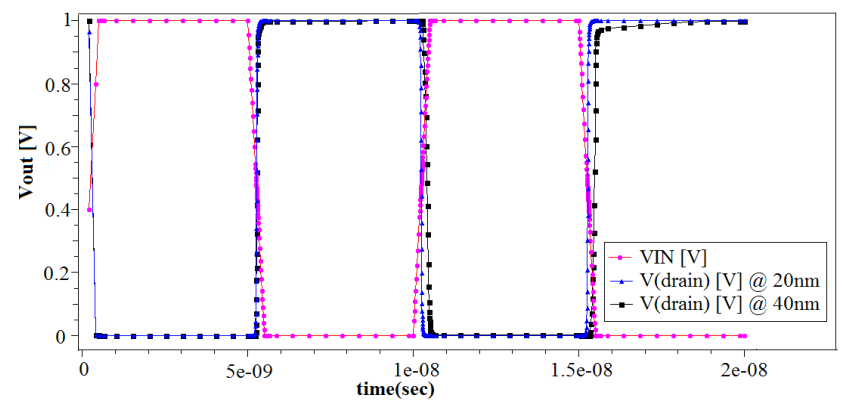

Figure 8. Transient behavior of double gate junction less CMOS inverter at channel lengths $20 \mathrm{~nm}$ and $40 \mathrm{~nm}$ of each JLT PMOS and JLT NMOS.

It can be illustrated from the figure that when channel lengths of both P-type and N-type JLT's is 20nm output response is taking lesser time than at channel lengths $40 \mathrm{~nm}$. This means that speed of operation at $20 \mathrm{~nm}$ is greater than $40 \mathrm{~nm}$. The blue line is reaching low or high potential first than the black line representing channel length $40 \mathrm{~nm}$. A better and an accurate transient curve of CMOS inverter can be obtained due to better performance parameters of Junction less transistors.

\section{Conclusion}

The double gate junction less transistor has proved to be better than conventional one in terms of performance parameters. The channel formation and the importance of both junction less and conventional transistors have been explained. Double gate junction less transistor based CMOS inverter is also structured and analysis for the same has been done at different technology nodes. It has been concluded that for scaled and smaller devices the junction less technology has proved to be capable of providing good performance of the devices with lesser flaws. The noise margin and speed of junction less CMOS inverter at various nodes have been discussed. Junction less based CMOS inverter resulted into sharp VTC pattern and can promise lesser fabrication steps than conventional inverter. Therefore at lower voltages, to overcome short channel effects, junction less based devices are best proven candidature for upcoming technology nodes. The device namely CMOS inverter made using junction less technology in this paper has proved that all characteristics of CMOS inverter is same that of conventional with little improvement, however in terms of fabrication point of view, for a fabrication engineer to make such devices with lower channel lengths is somewhere easier than conventional one.

\section{Acknowledgement}

Author would like to thank the faculty of Punjab University to provide all access to the labs and to provide all necessary help to complete this work presented in this paper.

\section{References}

1. Donald A. Neamen. Semiconductor Physics And Devices third edition. McGraw-hill.

2. International Technology Roadmap for Semiconductor.2008. Available http://public.itrs.net.

3. Moore GE. Cramming more components onto integrated circuits 1998 Proc. of the IEEE. 86(1) p. 82-5.

4. Colinge J, Lee C, Afzalian A, Akhavan N, Yan R, Ferain I, Razavi,B, O’Neill, Blake A, White, Kelleher AM, McCarthy $B$ and Murphy R. Nanowire transistors without junctions. Nat. Nanotechnology 2010; 5( 3):225-29.

5. Intekhab Amin S, Sarin R K, Junctionless Transistor A review. 432-39.

6. Colinge JP, Lee CW, Dehdashti Akhavan N, Yan R, Ferain I ,Razavi P, Kranti A Yu R. Junctionless Transistor:Physics And Properties. solid-state electron.2010. p. 33-7.

7. Gnani, Elena.et al. Theory of the Junctionless Nanowire FET. Electron devices.IEEE Transactions 2011: pp .2903910.

8. Lee C, Ferain I, Afzalian A,Yan R, Akhavan N, Razavi P, Colinge JP. Performance Estimation of Junctionless Multigate Transistors.Solid State Electron 2010; 54(2):97- 103.

9. Porag Jyoti Ligira, Gargi Khanna. Review on different types of junctionless transistor. International Journal of Emerging Technologies in Computational and Applied Sciences. 2014 . p. 404-8. 
10. Lee CW, Afzalian A, Akhavan ND, Colinge JP. Junctionless Multigate Field Effect Transistor. Applied Physics Letters. 2009; 94(5):053 511-2.

11. Juan Pablo Duarte,Sung-Jin Choi, Yang-Kyu Choi. A Full Range Drain Current Model For Double Gate Junctionless Transistors. IEEE Transactions on Electron Devices. 201158, no 12.

12. Ming-Hung Han,Chun-Yen Chang, Life Fellow,Hung-Bin Chen, Ya-chi Cheng Yang Chun. Device And Circuit Performance Estimation of Junctionless Bulk FinFet's IEEE Trans Electron Devices. 60. p. 1807-13.

13. Chaan-Hoon Park, Mayung-Dong Ko, Ki-Hyun Ki, RockHyun Beak, Chang-Woo Sohn, Chang Ki Baek, Sooyoung Park, M.j.Deen, Yoon-Ha Jeong, Jeong-Soo Lee. Electrical Characterstics of 20nm Junctionless Si Nanowire Transistor. Solid State Electronics. 2012: 73 . p.7-10.

14. Colinge JP ,Colinge CA. Physics of Semiconductor Devices, Kluwer Academic Publishers

15. Godoy A, Lopez-Villanueva JA, Jimenez-Tejada JA, Palm A, Gamiz F. A Simple Subthreshold Swing Model For Short Channel MOSFET's. Solid State Electronics. 2011. p. 39197.

16. Chi-Woo Lee, IsabelleFerain, Aryan Afzalian, Ran Yan, Nima Dehdashti Akhavan, Pedram Razavi, Jean-Pierre
Colinge. Performance Estimation of Junctionless Multigate Transistor Solid State Electronics. 2011. p. 97-103.

17. Prashanth Kumar B, Wasim Arif, Srimanta Baishya. An inclusive study on characterstics of junctionless transistor.

18. Colinge JP, Lee CW, Afzalian A, Dehdashti N, Yan R, Ferain I, Razavi P, B.,O'Nell, Blake A,White M, Kelleher A.M. SOI Gate resistor: CMOS Without Junctions IEEE, Int SOI Conference. 2009. p. 1-2.

19. Sung-Mo Kang, Yusuf Leblebici. CMOS Digital Integrated Circuits. Analysis and Design. Third edition.

20. Vishal Narula, Charu Narula, Jatinder Singh. Investigating Short Channel Effects and Performance Parameters of Double Gate Junction less Transistor at Various Technology Nodes RAECS IEEE. 2015.

21. Vishal Narula, Charu Narula, Jatinder Singh. Analysis and Comparison of Various Performance Parameters and Short Channel Effects of N Channel and P Channel Dual Gate Junction less Transistors At Different Technology Nodes. International Journal of Control Theory and Applications. 2016.

22. COGENDA TCAD Manual. 